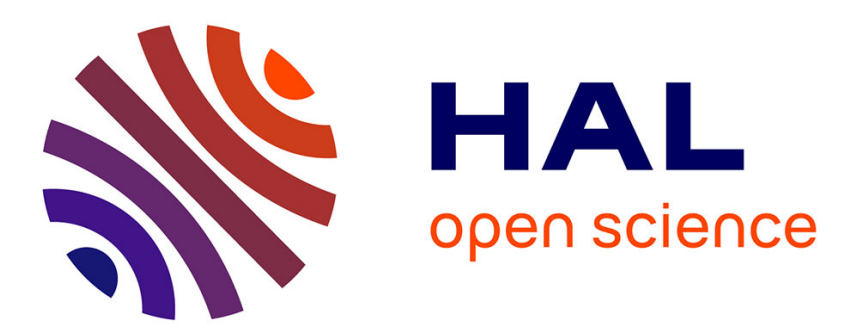

\title{
Étude par spectrométrie d'absorption infrarouge des dommages produits par des photons $\gamma$ et des ions lourds sur un triacétate de cellulose
}

\author{
J.P. Moliton, A. Barussaud, J.C. Vareille, J.L. Teyssier
}

\section{To cite this version:}

J.P. Moliton, A. Barussaud, J.C. Vareille, J.L. Teyssier. Étude par spectrométrie d'absorption infrarouge des dommages produits par des photons $\gamma$ et des ions lourds sur un triacétate de cellulose. Revue de Physique Appliquée, 1975, 10 (3), pp.109-115. 10.1051/rphysap:01975001003010900 . jpa00243888

\section{HAL Id: jpa-00243888 \\ https://hal.science/jpa-00243888}

Submitted on 1 Jan 1975

HAL is a multi-disciplinary open access archive for the deposit and dissemination of scientific research documents, whether they are published or not. The documents may come from teaching and research institutions in France or abroad, or from public or private research centers.
L'archive ouverte pluridisciplinaire HAL, est destinée au dépôt et à la diffusion de documents scientifiques de niveau recherche, publiés ou non, émanant des établissements d'enseignement et de recherche français ou étrangers, des laboratoires publics ou privés. 


\title{
ÉTUDE PAR SPECTROMÉTRIE D'ABSORPTION INFRAROUGE DES DOMMAGES PRODUITS PAR DES PHOTONS $\gamma$ ET DES IONS LOURDS SUR UN TRIACÉTATE DE CELLULOSE
}

\author{
J. P. MOliton, A. BARUSSAUd, J. C. VAREILle et J. L. TEYSSIER \\ Laboratoire des Radiations Ionisantes, \\ Université de Limoges, 123, rue Albert-Thomas, 87100 Limoges, France
}

(Reçu le 17 octobre 1974, accepté le 27 novembre 1974)

\begin{abstract}
Résumé. - Dans le cadre d'une étude du triacétate de cellulose en tant que détecteur solide visuel de traces, on montre par spectrométrie d'absorption infrarouge qu'à $20^{\circ} \mathrm{C}$ les photons $\gamma$ produisent surtout la formation de liaisons hydrogène entre chaînes cellulosiques voisines. Une préirradiation $\gamma$ provoque une amplification sensible de l'effet des ions lourds.Cela permet de supposer que les réactions primaires et secondaires induites par les ions lourds se produisent elles aussi entre chaînes cellulosiques voisines.
\end{abstract}

\begin{abstract}
In the framework of a study of cellulose triacetate as a Solid State Track Detector, we show by infrared techniques that in $\gamma$-irradiated cellulose triacetate films at $20^{\circ} \mathrm{C}$, hydrogen bonds between neighbouring cellulosic chains are mainly produced. $\gamma$ preirradiation creates a strong amplification of heavy ions effects. Therefore, primary and secondary reactions induced by heavy ions can be considered also as taking place between neighbouring cellulosic chains.
\end{abstract}

1. Introduction. - Dans certains dérivés cellulosiques, il est possible d'observer au microscope optique, après une attaque chimique appropriée, les traces d'ions lourds. Ces matériaux sont donc utilisés comme détecteurs solides visuels de traces sans que l'on connaisse pour autant les processus responsables de la formation de la trace. (Les effets des radiations U. V., $\mathrm{X}$, ou $\gamma$ sur ces types de polysaccharides sont d'ailleurs aussi mal connus.) De manière schématique, on peut supposer que la trace observée au microscope optique est créée en trois étapes :

- interaction directe de l'ion avec le matériau (réaction primaire) ;

- interaction entre le matériau irradié et le milieu ambiant (réaction secondaire) ;

- développement de la trace par attaque chimique des produits des réactions secondaires (réaction tertiaire).

Jusqu’à présent, on s'est intéressé le plus souvent aux phénomènes tertiaires, puisqu'on a la possibilité d'observer directement les traces développées. Pourtant le problème essentiel est de connaître la nature des produits des réactions primaires et secondaires qui constituent la trace latente. Il est possible d'obtenir des informations sur les premiers en supprimant les processus secondaires par refroidissement à basse température. On peut ensuite étudier la cinétique des réactions secondaires en ramenant progressivement les échantillons à la température ambiante [1]. Tout cela suppose qu'on ait au moins un dispositif qui permette le refroidissement des échantillons durant les irradiations en ions lourds sur accélérateur, ce qui n'est pas notre cas à l'heure actuelle. Nous avons donc fait appel à la spectroscopie d'absorption infrarouge qui nous a fourni des informations sur les produits des réactions secondaires après irradiation et conservation des échantillons à la température ambiante.

Nous avons étudié le comportement de films de triacétate de cellulose (T. A. C) contenant $10 \%$ de diéthyl-phtalate qui joue le rôle de plastifiant. Ces films, fabriqués au laboratoire, ont une épaisseur moyenne de $50 \mu \mathrm{m}$; ils sont répertoriés sous l'appellation $\mathbf{P}^{10}$. Ils sont irradiés successivement par les photons $\gamma \mathrm{du}{ }^{60} \mathrm{Co}$ et par des ions ${ }^{40} \mathrm{Ar}^{4+}$ de $1,1 \mathrm{MeV}$ par nucléon.

Une série de mesures préliminaires a montré qu'il fallait atteindre des doses supérieures au Mégarad pour que l'analyse infrarouge puisse déceler les dom- 
mages créés dans le matériau par le rayonnement gamma.

2. Dosimétrie gamma. - Nous disposions d'un appareil type «Jupiter Junior II BARAZZETTI» équipé d'une source de ${ }^{60} \mathrm{Co}$ dont l'activité était de $520 \mathrm{Ci}$ en mars 1971.

2. 1 ProblèmeS POSÉS PAR LE CHOIX DES DOSIMÈTRES. - Pour obtenir des doses élevées dans un temps raisonnable, le débit de dose doit être important. La seule manière de procéder est de se rapprocher de la source, c'est-à-dire d'irradier dans le collimateur et non à l'isocentre de l'appareil, comme c'est le cas habituellement. Le collimateur est constitué d'un assemblage de plaques de tungstène entrecroisées ; l'intérieur a la forme d'une pyramide tronquée à base carrée, dans le prolongement de laquelle se trouve la source. Deux types de problème se posent :

a) Il faut utiliser des dosimètres qui acceptent des débits de dose élevés et qui possèdent des dimensions compatibles avec celles du collimateur.

b) Les photons interéagissent avec les parois de tungstène. Au faisceau primaire s'ajoutent donc des photons dégradés en énergie et des électrons. Il est difficile de connaître l'importance des modifications apportées ainsi au spectre énergétique dans la mesure où une spectrométrie directe n'est pas réalisable.

2.2 Les SOLUTIONS UTILISÉES. - Les dosimètres ont été entourés d'une enveloppe de plexiglass qui assure l'équilibre électronique.

Pour avoir une idée de la dégradation du spectre énergétique à l'intérieur du collimateur, nous avons employé trois dosimètres dont les réponses en énergie sont différentes et étudié l'évolution de leur réponse en fonction de la distance à la source. Notre choix s'est porté sur les trois dosimètres suivants :

- dosimètre à l'Alanine dont la réponse croît avec l'énergie ;

- dosimètre Verre au Cobalt dont la réponse décroît lorsque l'énergie augmente ;

- dosimètre de Fricke $\left(\mathrm{FeSO}_{4}\right)$ dont la réponse est indépendante de l'énergie.

Deux remarques s'imposent :

a) Chaque dosimètre a une réponse pratiquement indépendante du débit de dose, donc de la distance à la source dans la gamme d'énergie considérée.

b) Par contre, ces dosimètres possèdent des réponses différentes entre eux. L'écart est de :

$6 \%$ entre les verres au Cobalt et le dosimètre de Fricke.

$10 \%$ entre l'alanine et le dosimètre de Fricke.

$1 \%$ entre le dosimètre de Fricke et une sonde du type Baldwin.
Pour rendre les mesures indépendantes de ces écarts, nous avons choisi pour étudier la réponse en fonction de la distance $x$ à la source, le paramètre $K(x)$ :

$$
K(x)=\frac{D_{\mathrm{d}}^{0}(x)}{D_{\mathrm{d}}^{0}(50)}
$$

avec

$$
\begin{aligned}
D_{\mathrm{d}}^{0}(x)= & \text { débit de dose mesuré par le dosimètre à } \\
& \text { la distance } x ; \\
D_{\mathrm{d}}^{0}(50)= & \text { débit de dose mesuré par le dosimètre à } \\
& 50 \mathrm{~cm} \text { de la source (isocentre). }
\end{aligned}
$$

La figure 1 montre que la variation de $K(x)$ est indépendante du dosimètre utilisé. Autrement dit, l'énergie moyenne du faisceau global ne varie pas de façon sensible avec la distance à la source. Dans ces conditions, il est possible d'effectuer des irradiations, d'énergie connue, à débit de dose relativement élevé en travaillant à l'intérieur du collimateur.

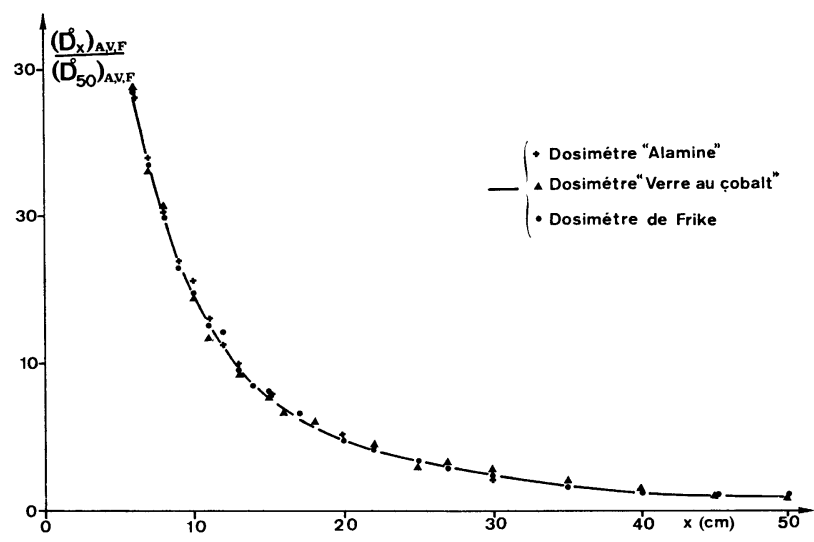

Fig. 1. - Courbe d'évolution de $K(x)$ pour les trois dosimètres utilisés.

3. Irradiation de films $\mathbf{P}^{10}$ par des photons $\gamma_{0}$ 3.1 Conditions D'IRRADIATION. - Toujours pour des questions d'équilibre électronique, les films ont été placés lors de l'irradiation dans un bloc de plexiglass identique à celui des dosimètres. Au bout d'un temps égal à la moitié de la durée d'irradiation, les films ont été retournés. Les irradiations ont eu lieu en deux périodes espacées de 4 mois environ. Cela peut poser un problème pour la première série d'échantillons (répertoriés de 1 à 6) dans la mesure où l'on ignore s'il se produit un effacement progressif de la dose $\gamma$ au cours du temps.

3.2 Calcul De LA DOSE ABSORbÉE. - La dose absorbée dans le triacétate de cellulose s'obtient à partir de la relation :

$$
D_{\mathrm{T} . \text { A.C. }}=D_{\mathrm{d}} \cdot \frac{(\mu / \rho)_{\mathrm{T} . \mathrm{A} . \mathrm{C}}}{(\mu / \rho)_{\mathrm{d}}}
$$


où $D_{\mathrm{d}}$ est la dose absorbée par le dosimètre et $(\mu / \rho)_{\mathrm{d}}$ son coefficient massique d'absorption. La valeur de

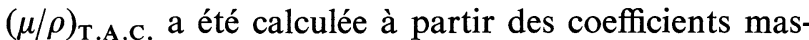
siques d'absorption des éléments carbone, hydrogène et oxygène qui constituent le triacétate de cellulose. Elle vaut $0,0296 \mathrm{~cm}^{2} / \mathrm{g}$. Les échantillons ont été irradiés à $6 \mathrm{~cm}$ de la source avec un débit de dose :

$$
D_{\mathrm{d}}^{0}(6)=0,039 \mathrm{Mrad} / \mathrm{h} .
$$

(Nous précisons cette valeur car l'influence du débit de dose sur les dommages créés n'est pas connue.) Moyennant quoi, les doses totales absorbées sont indiquées dans le tableau I, [2].

\section{TABLEAU I}

Valeurs des différentes doses de photons $\gamma$ pour des échantillons de films $\mathbf{P}^{10}$

\begin{tabular}{|c|c|c|c|c|c|c|c|c|c|c|c|c|}
\hline Dose $\mathrm{n}^{\circ}$ & 1 & 2 & 3 & 4 & 5 & 6 & 7 & 8 & 9 & 10 & 11 & 12 \\
\hline Mrads & 1,9 & 3,8 & 5,7 & 7,6 & 9,5 & 11,4 & 13,8 & 15,7 & 17,6 & 19,5 & 21,4 & 23,3 \\
\hline
\end{tabular}

3.3 Les RÉSUltats DE L'ANALYSE GLOBALE PAR SPECTROMÉTRIE D'ABSORPTION I. R. - Les spectres d'absorption, enregistrés sur un appareil Perkin-Elmer, modèle 225, avant et après irradiation gamma (Fig. 2 et 3), montrent qu'il n'y a en règle générale ni apparition ni disparition complète de bande. Toutefois la bande à $1162 \mathrm{~cm}^{-1}$ disparaît totalement pour une dose supérieure à $22 \mathrm{Mrad}$.

L'identification des bandes, qui a été traitée en détail dans un travail antérieur [3], ne pose pas de problème nouveau ; la situation est résumée par le tableau II.

Le tableau III indique, lui, l'évolution de différentes bandes en fonction de la dose $\gamma$. Le fait essentiel est que l'intensité du pied commun des bandes $v(\mathrm{O}-\mathrm{H})$ et $v(\mathrm{C}-\mathrm{H})$ à $3300 \mathrm{~cm}^{-1}$ varie de manière significative (Fig. 4). Si l'on trace la courbe de variation de

$$
d=\log \left(\frac{I_{0}}{I}\right)
$$

où :

$I_{0}$ est l'intensité avant l'irradiation à $3300 \mathrm{~cm}^{-1}$; $I$ est l'intensité après l'irradiation à $3300 \mathrm{~cm}^{-1}$

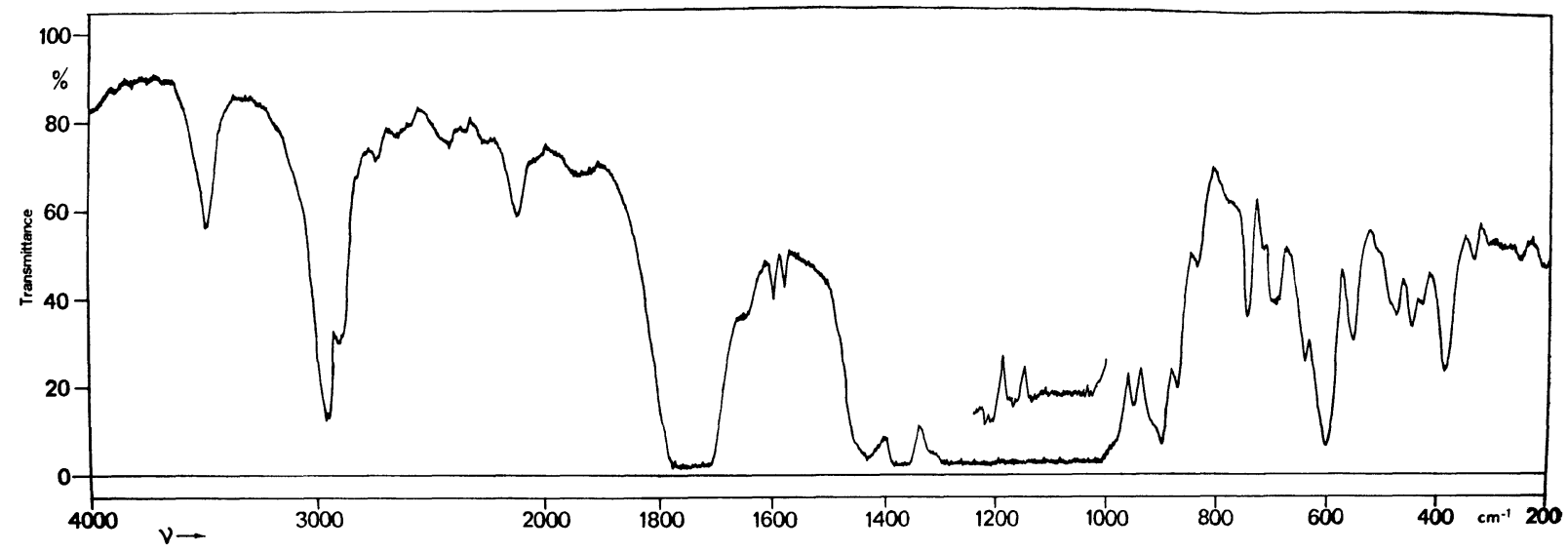

FIG. 2. - Spectre I. R. d'un film type $\mathbf{P}^{10}$ vierge.

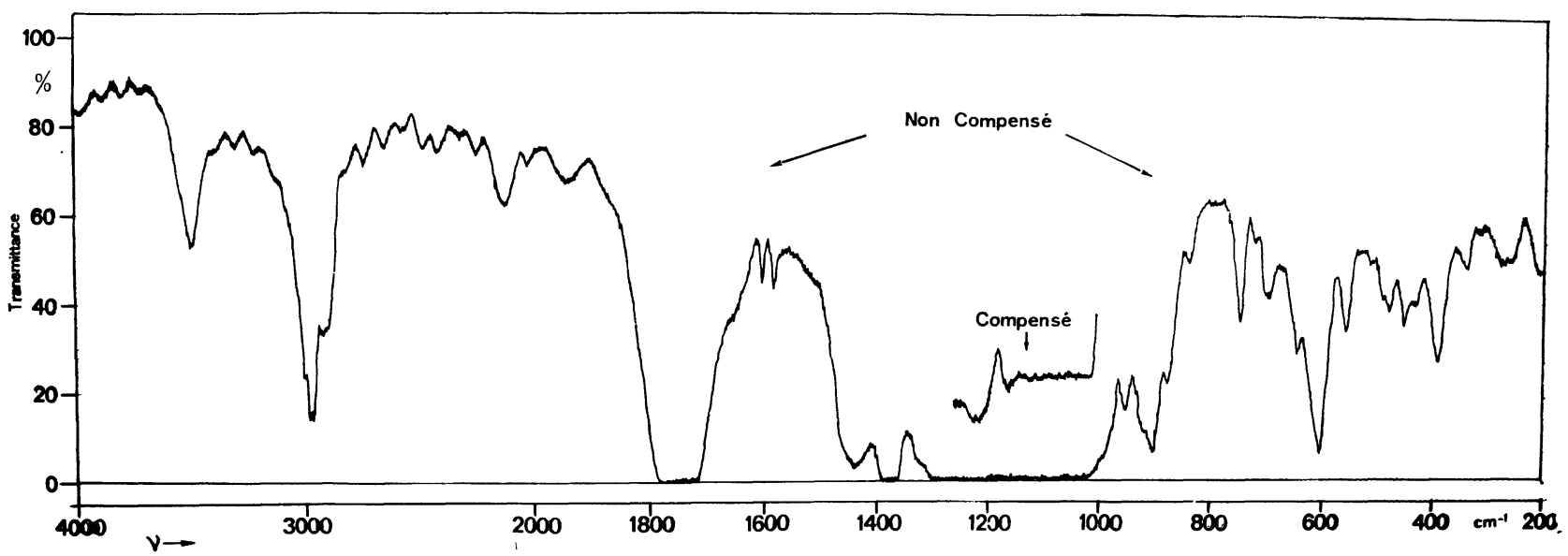

FIG. 3. - Spectre I. R. d'un film type $\mathbf{P}^{10}$ ayant reçu une dose de $17,6 \mathrm{Mrad}$ en photons $\gamma$. 


\section{TABLEAU II}

Identification et attribution des bandes d'absorption dans le domaine $5000-200 \mathrm{~cm}^{-1}$

\begin{tabular}{|c|c|}
\hline Nombre d'onde $\mathrm{cm}^{-1}$ & Attribution \\
\hline $3490-3500$ & $\begin{array}{l}\partial(O-H) \\
\text { dans tous les cotons et dérivés cellu losiques }\end{array}$ \\
\hline 2960 & $\partial(C-H)$ \\
\hline 1750 & $\begin{array}{l}\partial(C=0) \\
\text { du radical acétyle }\end{array}$ \\
\hline 1430 & $\begin{array}{l}\delta\left(\mathrm{CH}_{2}\right) \\
\text { balancement des hydrogènes du radical acé- } \\
\text { - tyle; bande couplée avec celle à } 899 \mathrm{~cm}^{-1}\end{array}$ \\
\hline 1370 & $\begin{array}{l}\delta\left(\mathrm{C}-\mathrm{CH}_{3}\right) \\
\text { du radical acétyle }\end{array}$ \\
\hline 1320 & $\delta\left(\mathrm{CH}_{2}\right)$ ou $\delta(\mathrm{OH})$ \\
\hline $1220-1240$ & $\begin{array}{l}\partial(C-0) \\
\text { du radical acétyle }\end{array}$ \\
\hline $1162(1120,1050,1036)$ & $\mathbf{C}_{11}-\mathbf{O}_{9}-\mathbf{C}_{7}-\mathbf{O}_{6}-\mathrm{C}_{4}$ \\
\hline 948 & Type 1 des sucres \\
\hline 899 & $\begin{array}{l}\delta\left(\mathrm{CH}_{2}\right) \\
\text { rotation plane des hydrogènes des carbo- } \\
\text {-nes(6);bande couplée avec celle à } \\
1430 \mathrm{~cm}^{-1}\end{array}$ \\
\hline 872 & Type 2 des sucres \\
\hline $\begin{array}{ll}835 & 490 \\
690 & 470 \\
640 & 445 \\
600 & 437 \\
550 & 390 \\
525 & 395\end{array}$ & Non identifièes \\
\hline
\end{tabular}

\section{TABLEAU III}

Evolution de l'intensité des bandes pour des films $\mathbf{P}^{10}$ irradiés en photons $\gamma$

\begin{tabular}{|c|c|c|}
\hline $\begin{array}{c}\text { Nombre } \\
\text { donde } \mathrm{cm}^{-1}\end{array}$ & Bande & Résultat \\
\hline 3500 & $\partial(O-H)$ & $\begin{array}{l}\text { lintensité croit jusqu'à un facteur } 1,5 \text { avec } \\
\text { déformation de son pied commun avec la } \\
\text { bande } J(C-H) \text { pour une dose de } 23 \mathrm{Mrads}\end{array}$ \\
\hline $\begin{array}{l}3000 \\
2960\end{array}$ & $\partial(\mathbf{C}-\mathbf{H})$ & statuquo \\
\hline 1162 & $\mathbf{C}-\mathbf{O}-\mathbf{C}-\mathbf{O}-\mathbf{C}$ & $\begin{array}{l}\text { décroît jusqu'à un facteur } 0,3 \text { pour une } \\
\text { dose de } 23 \text { Mrads }\end{array}$ \\
\hline 948 & cycle pyran & $\begin{array}{l}\text { décroît jusqu'à un facteur } 0,5 \text { pour une } \\
\text { dose de } 23 \text { Mrads }\end{array}$ \\
\hline 899 & $\delta\left(\mathrm{CH}_{2}\right)$ & statuquo \\
\hline $\begin{array}{l}1580 \\
1600\end{array}$ & $\begin{array}{l}\text { noyau phényle } \\
\text { du plastifiant }\end{array}$ & $\begin{array}{l}\text { décroît faiblement facteur } 0,7 \text { pour } \\
\text { une dose de } 23 \mathrm{Mrads}\end{array}$ \\
\hline 740 & $\delta(\mathrm{C}-\mathrm{H}) \varphi$ & $\begin{array}{l}\text { décroît faiblement facteur } 0,7 \text { pour } \\
\text { une dose de } 23 \text { Mrads }\end{array}$ \\
\hline
\end{tabular}

en fonction de la dose absorbée (Fig. 5), la réponse est linéaire. On a là la possibilité d'une mesure de doses entre 2 et $50 \mathrm{Mrad}$. La précision des mesures ne dépasse pas ici 1,25 Mrad car la valeur de $I_{0}$ a été mesurée sur un échantillon différent de celui qui a été irradié. Or, d'un échantillon à l'autre, il est courant d'enregistrer des variations d'épaisseur de $6 \%$. Si l'on prend la précaution de pointer $I_{0}$ sur le même échantillon, la précision est de 0,5 Mrad.

4. Postirradiations en ions ${ }^{40} \mathrm{Ar}^{4+}(1,1 \mathrm{MeV} /$ nucléon). - Les irradiations ont eu lieu au C. E. V. d'Orsay (films maintenus à $20^{\circ} \mathrm{C}$ et $10^{-8}$ torr). Nous faisons référence aux résultats obtenus dans un travail antérieur [3] en ce qui concerne les échantillons directement irradiés par des ions lourds.

Il s'agit d'ions $\mathrm{K}_{\mathrm{r}}^{8+}(1,1 \mathrm{MeV} /$ nucléon $)$. La comparaison avec les films irradiés successivement par des photons $\gamma$ et des ions $\mathrm{A}_{\mathrm{r}}^{4+}(1,1 \mathrm{MeV} /$ nucléon) est possible dans la mesure où les parcours des deux types d'ions sont très proches. Elle montre (Fig. 6 et 7) :

1) que la déformation de la bande $v(\mathrm{O}-\mathrm{H})$ et de son pied à $3300 \mathrm{~cm}^{-1}$ produite par les photons $\gamma$ subsiste après l'irradiation en ions lourds ;

2) que le profil du spectre dans le domaine 800$200 \mathrm{~cm}^{-1}$, qui est toujours très peu affecté par une irradiation en ions lourds [3], est raboté lorsqu'il y a eu préirradiation en photons $\gamma$;

3) que cet effet de rabotage dépend principalement de la dose $\gamma$, puisque pour une dose inférieure en photons $\gamma$ et supérieure en ions lourds, l'effet est moindre : figure $7\left(23 \mathrm{Mrad}\right.$ en $\gamma+12 \times 10^{12}$ ions $\left./ \mathrm{cm}^{2}\right)$ et figure $8\left(14 \mathrm{Mrad}\right.$ en $\left.\gamma+16 \times 10^{12} \mathrm{ions} / \mathrm{cm}^{2}\right)$.

5. Synthèse et interprétation. - La présente étude apporte les éléments d'information suivants dans le domaine de doses étudiées :

1) Différence fondamentale : les photons $\gamma$ ne provoquent aucune variation du fond continu contrairement aux ions lourds. Le film reste translucide, alors qu'il jaunit et devient opaque sous l'effet de ces derniers.

2) Mise à part la bande à $1162 \mathrm{~cm}^{-1}$, il n'y a pas de variation d'intensité importante, notamment pas de disparition de bande d'absorption due aux photons $\gamma$, par opposition aux ions lourds.

3) L'irradiation en ions lourds seuls ne déforme pas le pied de la bande $v(\mathrm{O}-\mathrm{H})$ comme le fait une irradiation en photons $\gamma$.

4) L'effet de rabotage dans le domaine $800-200 \mathrm{~cm}^{-1}$ produit par le rayonnement $\gamma$ montre qu'il ne reste de la molécule de T. A. C., après une irradiation en ions lourds, que les liaisons :

$$
\begin{aligned}
& \mathrm{O}-\mathrm{H} \\
& \mathrm{C}-\mathrm{H} \\
& \mathrm{C}=\mathrm{O}
\end{aligned}
$$



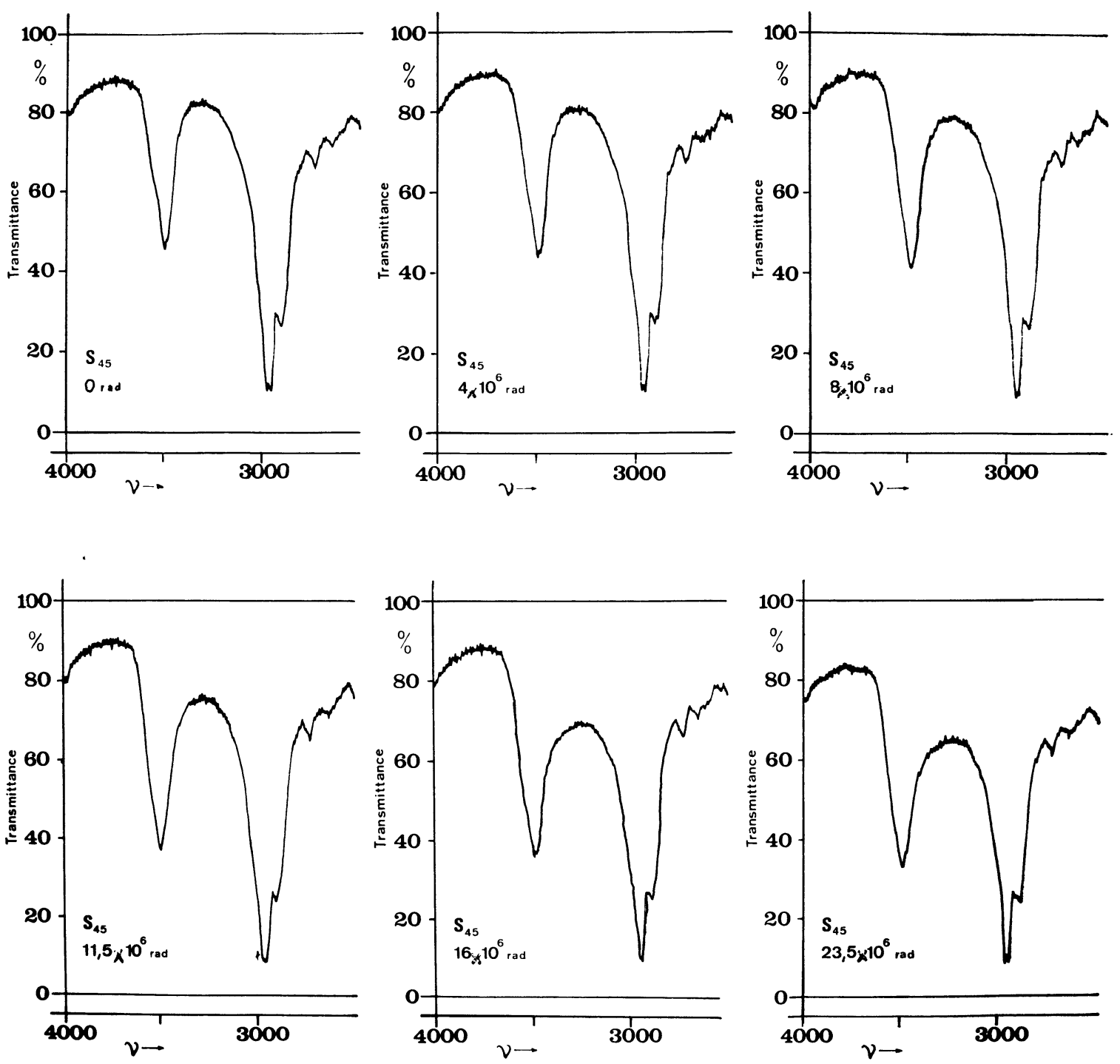

Fig. 4. - Evolution, en fonction de la dose $\gamma$, du pied à $3300 \mathrm{~cm}^{-1}$ de la bande $v(\mathrm{O}-\mathrm{H})$.

sans aucune bande d'absorption caractéristique d'une structure quelconque. Sous l'effet conjugué des deux radiations, l'organisation macromoléculaire existant dans le T. A. C. semble avoir été détruite, alors que chacune de ces radiations, prise séparément, ne perturbe pas l'édifice de façon aussi importante.

5) L'hypothèse de la formation d'un cycle furannique [4] ne peut être retenue : aucune bande caractéristique de cette structure n'apparaît. Toute interprétation repose sur la signification que l'on peut accorder à l'effet prépondérant des photons $\gamma$, c'est-à-dire la modification d'intensité du pied à $3300 \mathrm{~cm}^{-1}$ de la bande $v(\mathrm{O}-\mathrm{H})$. Nous avons retenu deux hypothèses :

1) l'existence d'un ou plusieurs radicaux libres de longue durée de vie ;

2) la formation de liaisons type pont hydrogène.

Nous ne croyons pas à la validité de la première hypothèse car si Deffner et Paretzke [1] ont montré la production de radicaux libres dans le T.A.C. par irradiation gamma, c'est au cours de la réaction primaire. Toujours selon ces auteurs, la vitesse de disparition à $20^{\circ} \mathrm{C}$ de ces radicaux libres est de $21,4 \%$ par heure. Au bout d'un mois, la densité de présence en radicaux libres ne nous paraît pas suffisante pour donner un signal décelable en spectroscopie infrarouge.

La seconde hypothèse, par contre, peut être étayée par plusieurs arguments. D'abord, les liaisons par pont hydrogène se manifestent toujours à des fréquences plus basses que la liaison $(\mathrm{O}-\mathrm{H})$ classique. Ensuite, Rowen [5] a montré que l'apparition du pont hydrogène se manifeste par la déformation du pied à 3250 $3300 \mathrm{~cm}^{-1}$ de la bande $v(\mathbf{O}-\mathbf{H})$, dans la cellulose et ses dérivés. Rowen a supposé que le pont ne se trouvait pas à l'intérieur d'une même chaîne cellulosique, mais entre 


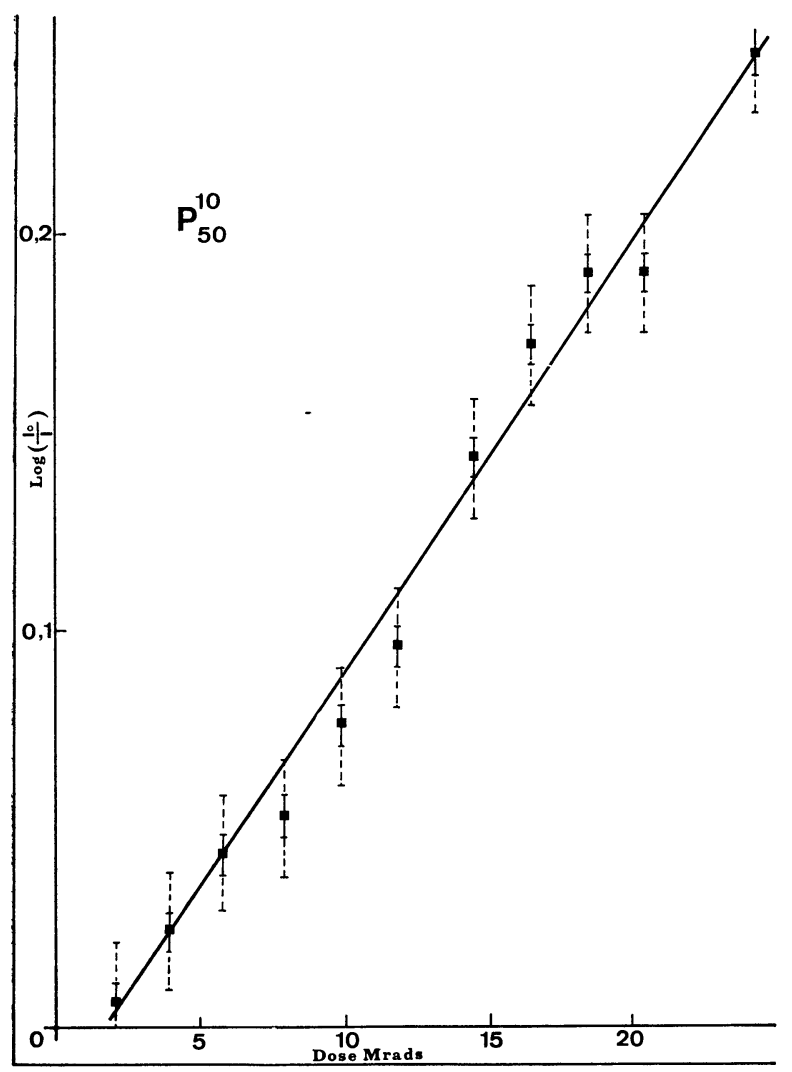

chaînes voisines. Cela expliquerait notamment que l'on n'obtienne pas une bande caractéristique d'un vibrateur $(\mathrm{O}-\mathrm{H})$, mais un massif d'absorption dans la zone des $3300 \mathrm{~cm}^{-1}$. La cellulose est en effet constituée par la juxtaposition de zones cristallines et amorphes qui s'interpénètrent. Plusieurs types de pontages $(\mathrm{O}-\mathrm{H})$ sont alors possibles. Il n'existe pas une valeur de la constante de rappel du vibrateur $(\mathrm{O}-\mathrm{H})$, mais plusieurs ; par conséquent on peut s'attendre à ce qu'il n'y ait pas de fréquence caractéristique. Dernier point, le pontage entre chaînes a normalement pour effet d'augmenter la dureté du matériau. Nous avons constaté que plus la dose gamma est élevée, plus les films de T. A. C. sont cassants. Compte tenu du fait que la préirradiation $\gamma$ augmente l'effet des ions lourds dans une proportion que l'énergie déposée ne laisse pas prévoir, les réactions primaires et secondaires induites par ions doivent se produire entre chaînes cellulosiques voisines et non à l'intérieur d'une même chaîne. C'est le rapprochement des chaînes effectué par les ponts hydrogène qui semble responsable de l'augmentation des dommages créés par les ions.

$\leftarrow$ Fig. 5. - Variation de $d=\log \left(I_{0} / I\right)$ à $3300 \mathrm{~cm}^{-1}$ en fonction de la dose $\gamma$.

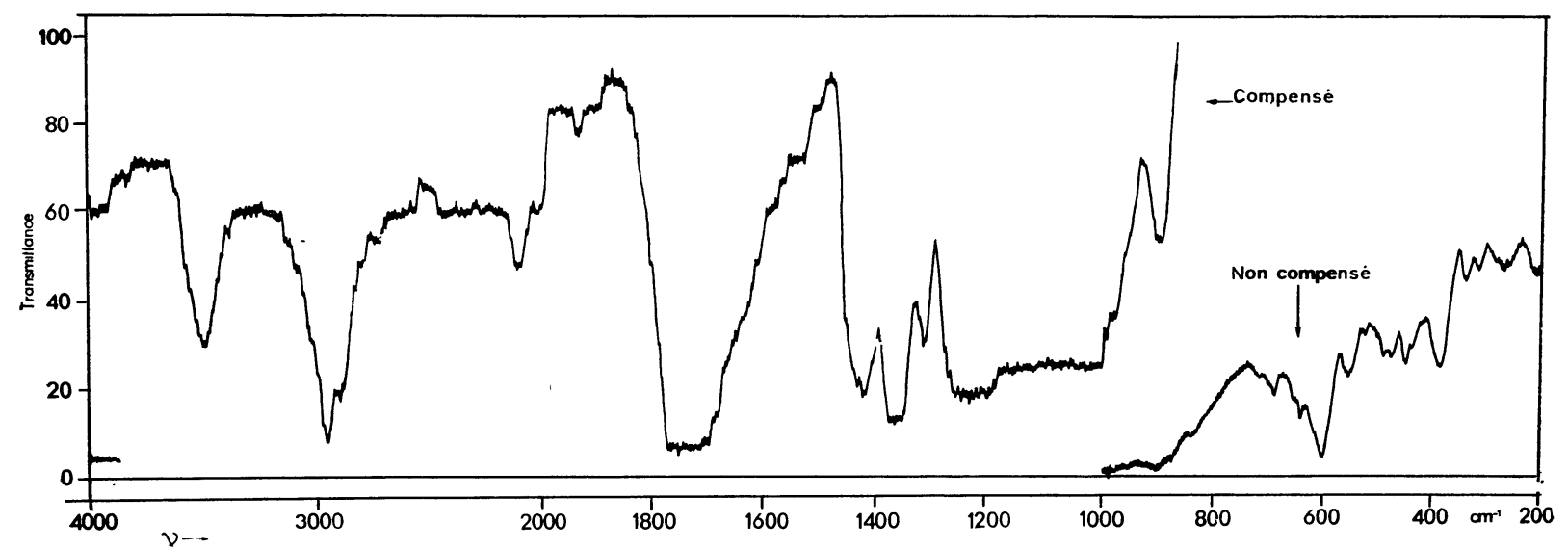

Fig. 6. - Spectre d'un échantillon $\mathrm{P}^{10}$ ayant reçu $9,2 \times 10^{12}$ ions $\mathrm{K}_{\mathrm{r}}^{8+}(1,1 \mathrm{MeV} / \mathrm{uma}) / \mathrm{cm}^{2}$.

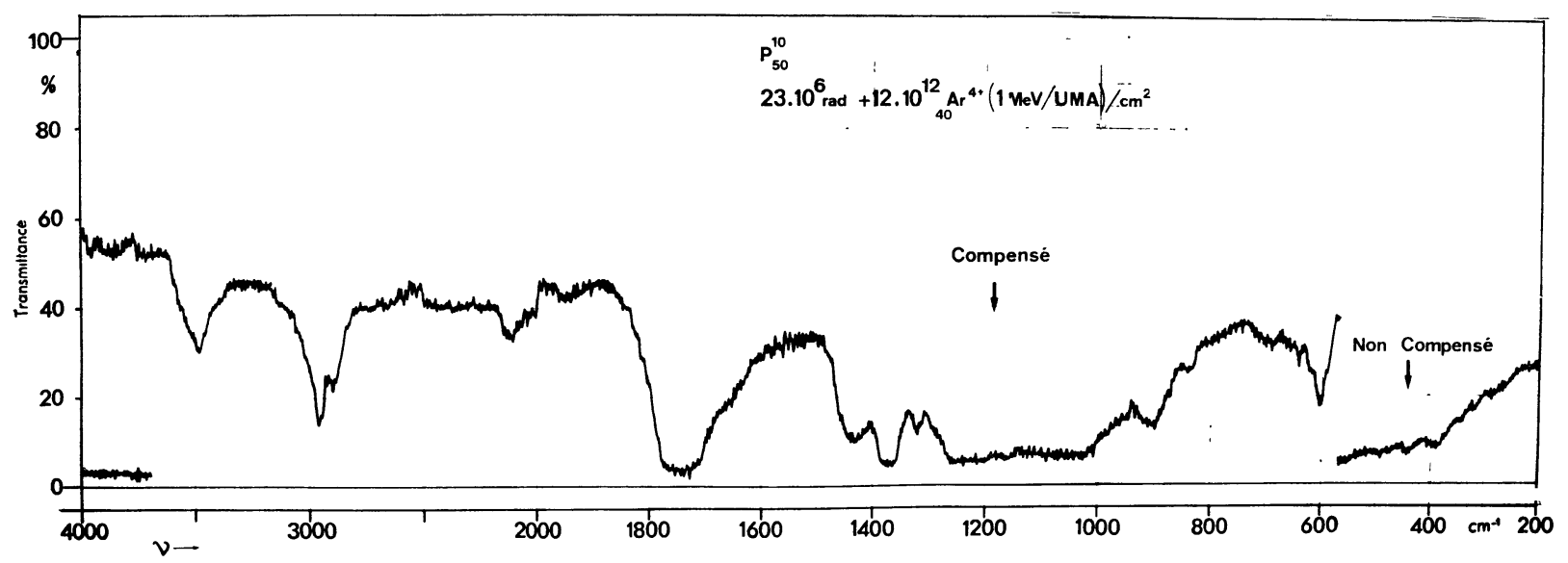

Fig. 7. - Spectre d'un échantillon $\mathrm{P}^{10}$ ayant reçu successivement une dose de $23 \mathrm{Mrad}$ en photons $\gamma$ et $12 \times 10^{12}$ ions $\mathrm{Ar}^{4+}$ $(1,1 \mathrm{MeV} / \mathrm{uma}) / \mathrm{cm}^{2}$. 


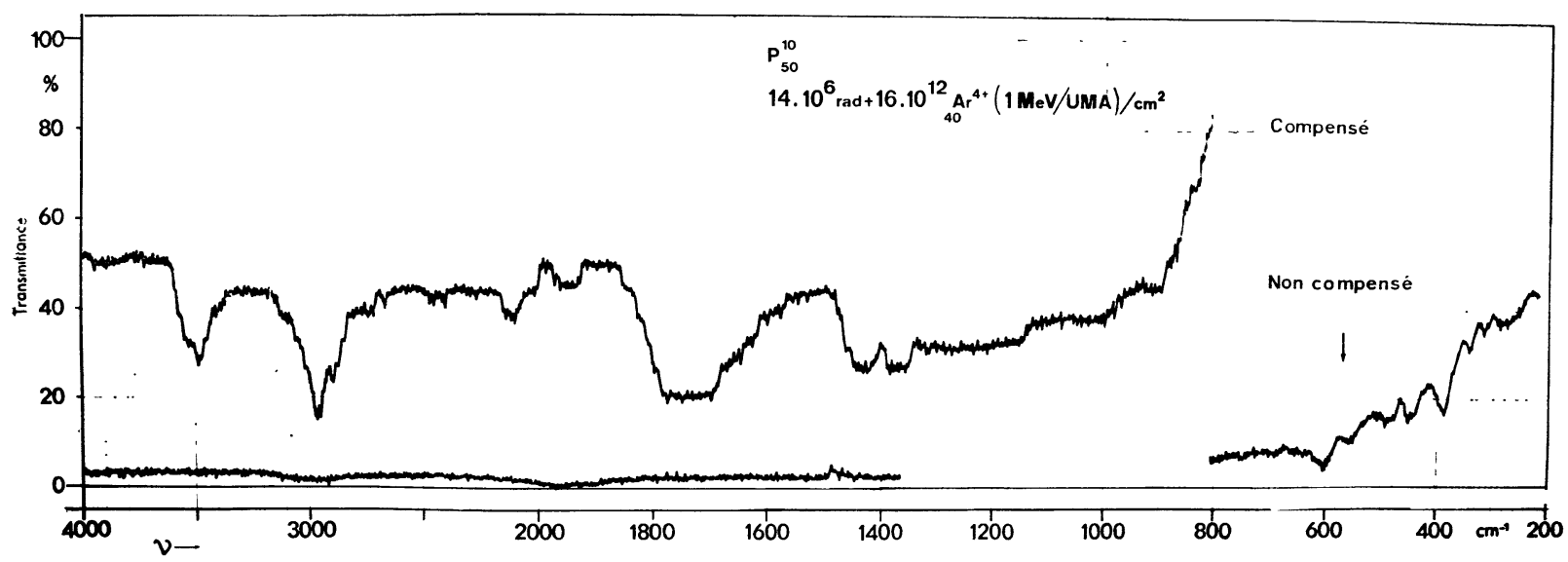

Fig. 8. - Spectre d'un film $\mathrm{P}^{10}$ ayant reçu successivement une dose de $14 \mathrm{Mrad}$ en photons $\gamma$ et $16 \times 10^{12}$ ions $\mathrm{Ar}^{4+}$ $(1,1 \mathrm{MeV} / \mathrm{uma}) / \mathrm{cm}^{2}$.

6. Conclusion. - En ce qui concerne l'action des ions lourds, nous savions [3] que le résultat des réactions primaires et secondaires était la coupure des trois types de liaison $(\mathrm{C}-\mathrm{O})$ que contient le monomère de T. A. C. et cela par pas de dose de $2 \times 10^{12}$ ions $/ \mathrm{cm}^{2}$, avec un certain ordre. Les nouveaux éléments d'information que nous possédons maintenant laissent penser que ces réactions et principalement celles d'élimination au stade secondaire, mettent en jeu au moins deux chaînes cellulosiques voisines. Sans doute faut-il abandonner l'idée d'un mécanisme réactionnel cyclique opérant sur un monomère.
Une autre conclusion résulte de la comparaison des deux types d'interaction, ions lourds et photons gamma : les ions lourds engendrent des réactions chimiques importantes (coupure des liaisons $\mathrm{C}-\mathrm{O}$ ) ; les photons $\gamma$ créent principalement des liaisons hydrogène, tout au moins jusqu'à $23 \mathrm{Mrad}$ et provoquent la coupure d'une seule liaison $\mathrm{C}-\mathrm{O}$ : celle du pont entre monomères à $1162 \mathrm{~cm}^{-1}$.

Remerciements. - Nos remerciements vont au Laboratoire de Physique Moléculaire de notre Université qui nous a permis de réaliser les expériences de spectrographie infrarouge.

\section{Bibliographie}

[1] Deffner, U. et Paretzke, H., Radiation Research 49 (1972) 272-77.

[2] Barussaud, A., Thèse de spécialité. Université Paul-Sabatier, Toulouse (1974).

[3] Moliton, J. P., Vareille, J. C. et Teyssier, J. L., Revue Phys. Appl. 9 (1974) 731-741.
[4] Rozmarin, Gh. et Butnaru, R., Cellulose Chem. Technol. 6 (1972) 393-404.

[5] Rowen, J. et Phyler, E. K., Natl. Bur. Stand. 44 (1950) 313-320. 